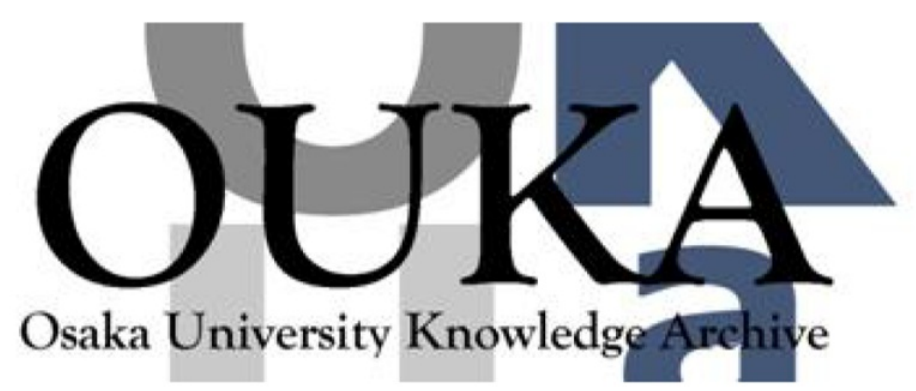

\begin{tabular}{|c|l|}
\hline Title & $\begin{array}{l}\text { Studies of ultra-intense laser plasma } \\
\text { interactions for fast ignition }\end{array}$ \\
\hline Author(s) & Tanaka, K. A.; Kodama, R.; Fujita, H. et al. \\
\hline Citation & Physics of Plasmas. 7(5) p. 2014-p. 2022 \\
\hline Issue Date & $2000-05$ \\
\hline oaire:version & VoR \\
\hline URL & https://hdl. handle. net/11094/3379 \\
\hline rights & \\
\hline Note & \\
\hline
\end{tabular}

Osaka University Knowledge Archive : OUKA

https://ir. Library. osaka-u. ac. jp/

Osaka University 


\title{
Studies of ultra-intense laser plasma interactions for fast ignition*
}

\author{
K. A. Tanaka, ${ }^{\dagger, a)}$ R. Kodama, H. Fujita, M. Heya, N. Izumi, Y. Kato, ${ }^{\text {b) }}$ \\ Y. Kitagawa, K. Mima, N. Miyanaga, T. Norimatsu, A. Pukhov, ${ }^{c}$ A. Sunahara, K. \\ Takahashi, ${ }^{\text {b) }}$ M. Allen, H. Habara, T. Iwatani, T. Matusita, T. Miyakosi, M. Mori, \\ H. Setoguchi, T. Sonomoto, M. Tanpo, S. Tohyama, H. Azuma, T. Kawasaki, \\ T. Komeno, O. Maekawa, S. Matsuo, T. Shozaki, Ka Suzuki, H. Yoshida, \\ and T. Yamanaka \\ Institute of Laser Engineering, Osaka University, Suita, Osaka 565-0871 Japan \\ Y. Sentoku \\ Institute for Laser Technology, Utubo-Honmachi 1-8-4, Osaka 550-0004 Japan \\ F. Weber, T. W. Barbee, Jr., and L. DaSilva \\ Lawrence Livermore National Laboratory, Livermore, California 94550
}

(Received 16 November 1999; accepted 4 February 2000)

\begin{abstract}
Laser plasma interactions in a relativistic parameter regime have been intensively investigated for studying the possibility of fast ignition in inertial confinement fusion (ICF). Using ultra-intense laser systems and particle-in-cell (PIC) simulation codes, relativistic laser light self-focusing, super hot electrons, ions, and neutron production, are studied. The experiments are performed with ultra-intense laser with $50 \mathrm{~J}$ energy, $0.5-1 \mathrm{ps}$ pulse at $1053 \mathrm{~nm}$ laser wavelength at a laser intensity of $10^{19} \mathrm{~W} / \mathrm{cm}^{2}$. Most of the laser shots are studied under preformed plasma conditions with a 100 $\mu \mathrm{m}$ plasma scale length condition. In the study of laser pulse behavior in the preformed plasmas, a special mode has been observed which penetrated the preformed plasma all the way very close to the original planar target surface. On these shots, super hot electrons have been observed with its energy peak exceeding $1 \mathrm{MeV}$. The energy transport of the hot electrons has been studied with making use of $K \alpha$ emissions from a seeded metal layer in planar targets. The details of ion acceleration followed by beam fusion reaction have been studied with neutron spectrometers. Laser ponderomotive force self-focusing and hot electron generation have been applied to a compressed core to see the effect of heating by injecting 12 beams of $100 \mathrm{ps}, 1 \mathrm{TW}$ pulses. (C) 2000 American Institute of Physics. [S1070-664X(00)95605-2]
\end{abstract}

\section{INTRODUCTION}

The concept of fast ignition (FI) (Refs. 1 and 2) is to inject an ultra-intense short laser pulse(s) into a highly compressed, high density fuel core within the core disassembling time, making use of relativistic laser self-focusing or guided channel formation. The ignition may be performed with the use of hot electrons or energetic ions, whose temperatures are of the order of $\mathrm{MeV}$, much higher than the thermal temperature of the plasmas. The attractive points of the concept are possibly to obtain fusion gains much higher and requiring laser irradiation uniformity less than those in the central spark scheme in inertial confinement fusion (ICF). The chirped pulse amplification method ${ }^{3}$ made it possible to construct ultra-intense laser systems with $100 \mathrm{TW}$ to PW peak intensities. Laser plasma interactions using these laser systems have opened new research in relativistic laser plasma

\footnotetext{
*Paper FI2 5 Bull. Am. Phys. Soc. 44, 93 (1999).

$\dagger$ Invited speaker.

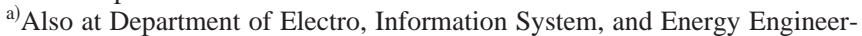
ing, Osaka University, Suita, Osaka 565-0871 Japan.

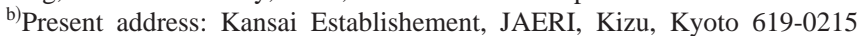
Japan.

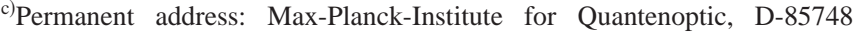
Garching, Germany.
}

interactions. ${ }^{4-6}$ The new experimental possibilities can be applied also to many other fields, such as particle acceleration, medical and industrial radiography, x-ray laser, and laboratory astrophysics.

We have studied the ultra-intense laser plasma interactions related to fast ignitor in ICF using our 50-100 TW laser systems and particle-in-cell (PIC) simulation codes. Among the issues to be studied, we focused on the laser beam behavior in plasmas, super hot electron generation, energetic ion production, and subsequent beam fusion process. As the second set of experiments, laser ponderomotive force self-focusing ${ }^{7,9}$ and hot electron generation have been applied to a compressed core to see the effect of heating by injecting 12 beams of $100 \mathrm{ps}, 1 \mathrm{TW}$ pulses in order to model the fast ignition scheme.

\section{EXPERIMENTAL FACILITIES}

Experiments were conduced using the 100 TW laser [peta watt module (PWM)] line coupled with the GEKKO XII laser system. With the conventional chirped pulse amplification (CPA) technique, the pulse in the PWM can deliver a $50 \mathrm{~J}$ with a $0.5-1 \mathrm{ps}$ pulse at a $1 \mu \mathrm{m}$ laser wavelength. Twenty percent of the ultra-short pulse energy can be focused to a spot size of $10 \mu \mathrm{m}$ full-width at half-maximum (FWHM) using an F/3.5 on-axis parabola at a vacuum inten- 
(a)

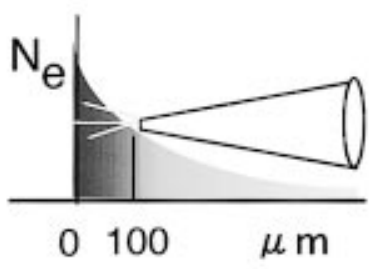

(b)

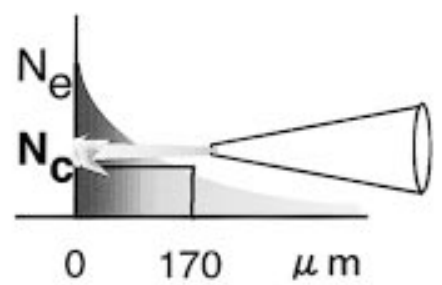

(c)

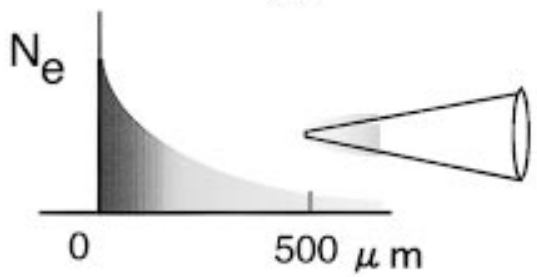

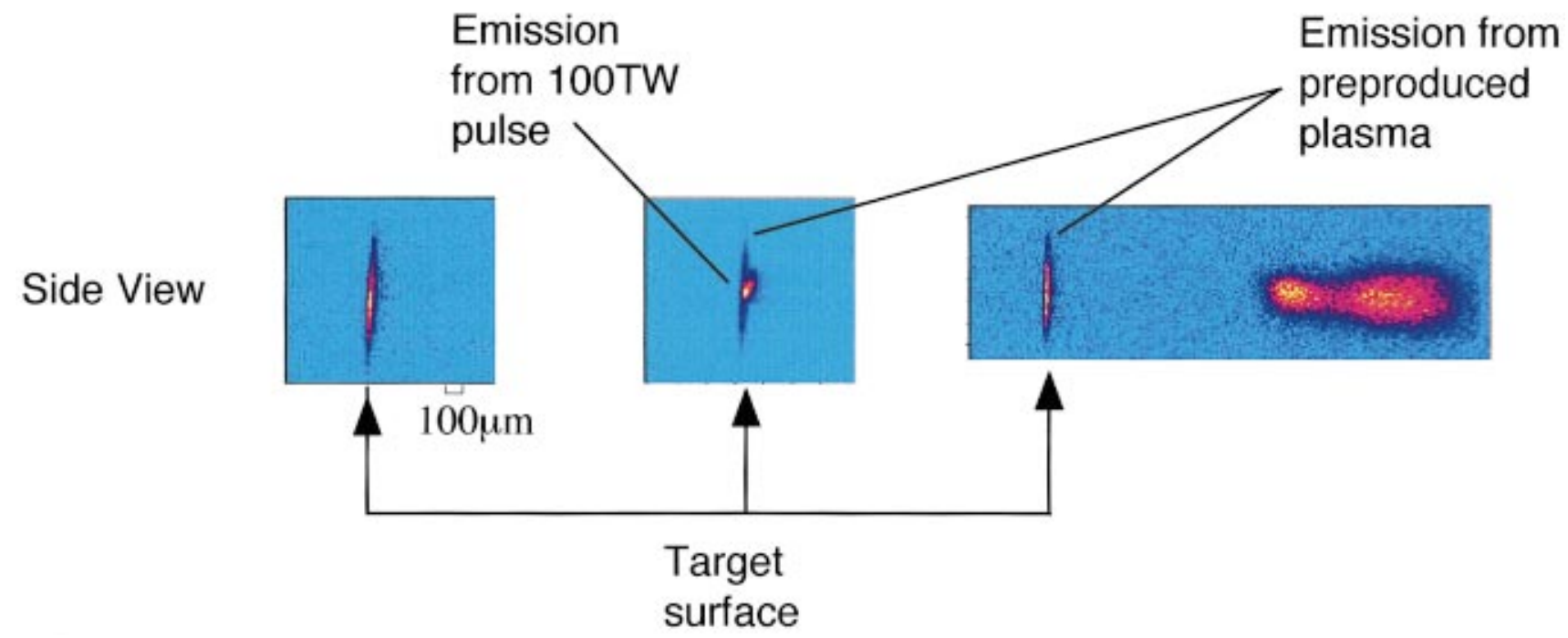

FIG. 1. (Color) X-ray pinhole pictures of ultra-intense laser shot into a plasma (side-on view). The focus point was set at (a) $100 \mu \mathrm{m}$, (b) $170 \mu \mathrm{m}$, and (c) $500 \mu \mathrm{m}$ from the original planar target surface. A clear local emission on the target surface seen in (b) is due to the penetration of the ultra-intense laser pulse.

sity of $10^{18}-10^{19} \mathrm{~W} / \mathrm{cm}^{2}$. Preformed plasmas were created by focusing a 100 ps laser pulse of any beam of the GEKKO XII at the wavelengths either $1 \mu \mathrm{m}$ or $0.53 \mu \mathrm{m}$, or focusing a $1 \mathrm{~ns}$ partially coherent laser pulse at $1 \mu \mathrm{m}$ or $0.5 \mu \mathrm{m}$ within a $500 \mu \mathrm{m}$ spot size. All $12 \mathrm{arms}$ of the GEKKO XII can deliver partially coherent laser (PCL) pulses of $1 \mathrm{~ns}$ (square shape) with $100 \mathrm{~J} /$ pulse at $1 \mu \mathrm{m}$ followed by $100 \mathrm{ps}$ double pulses with $120 \mathrm{~J} /$ pulse energy at $1 \mu \mathrm{m}$. The PCL laser has a large divergence angle of $120 \mu \mathrm{rad}$, resulting in a $150 \mu \mathrm{m}$ diam in the far field spot. The $100 \mathrm{ps}$ double pulses have been used for hole boring ${ }^{7-9}$ a corona plasma by focusing tightly in front of the shell target surface.

\section{EXPERIMENTS AND RESULTS}

Our experiments related to fast ignitor have been conducted as follows. First, three categories are performed with an ultra-short pulse at $10^{19} \mathrm{~W} / \mathrm{cm}^{2}$ laser intensity. The last category is with a short pulse at $10^{17} \mathrm{~W} / \mathrm{cm}^{2}$.

Those are

(1) Ultra-intense laser behaviors in preformed plasmas;

(2) Hot electron generation and its heating of a solid target;

(3) Energetic ion generation and its subsequent fusion process; and

(4) First demonstration of a compressed core heated by short pulse self-focusing and hot electrons.

\section{A. Ultra-intense laser behaviors in preformed plasmas}

It is an important subject for the fast ignitor concept to know if an ultra-intense laser with an intensity exceeding $10^{19} \mathrm{~W} / \mathrm{cm}^{2}$ can penetrate a thick corona plasma with whole beam relativistic self-focusing. There are several works about relativistic self-focusing in plasmas, both theoretical simulation and experiments..$^{10,11}$ However, all the previous works dealt with the self-focusing with underdense plasmas or in a thin overdense plasma. It is more desirable for FI to study the details of relativistic self-focusing behaviors in a long exponential profile with a large overdense region. We have studied laser light behaviors in preproduced plasmas with a $100 \mu \mathrm{m}$ density scale length created on a solid planar plastic target. The density profile of the preproduced plasma along the laser axis had the critical density $\left(n_{c}\right)$ for $1 \mu \mathrm{m}$ laser light at $100 \mu \mathrm{m}$ from the target surface. The plasma is close to the ones used in our previous studies.

The experiments were carried out with the $\lambda=1.053 \mu \mathrm{m}$, 100 TW laser line synchronized with the GEKKO XII laser system within a time jitter of less than 100 ps. Thus any of the GEKKO XII laser system's 12 beams could be used to create preproduced plasma at $\lambda=0.53 \mu \mathrm{m}$, with a pulse width from $0.1 \mathrm{~ns}$.

A relativistic self-focusing experiment has been conducted changing the focal position of the $100 \mathrm{TW}$ beam along the laser axis relative to the preproduced plasma. ${ }^{12}$ The preproduced plasmas were created on planar CD (deuterated 
(a)
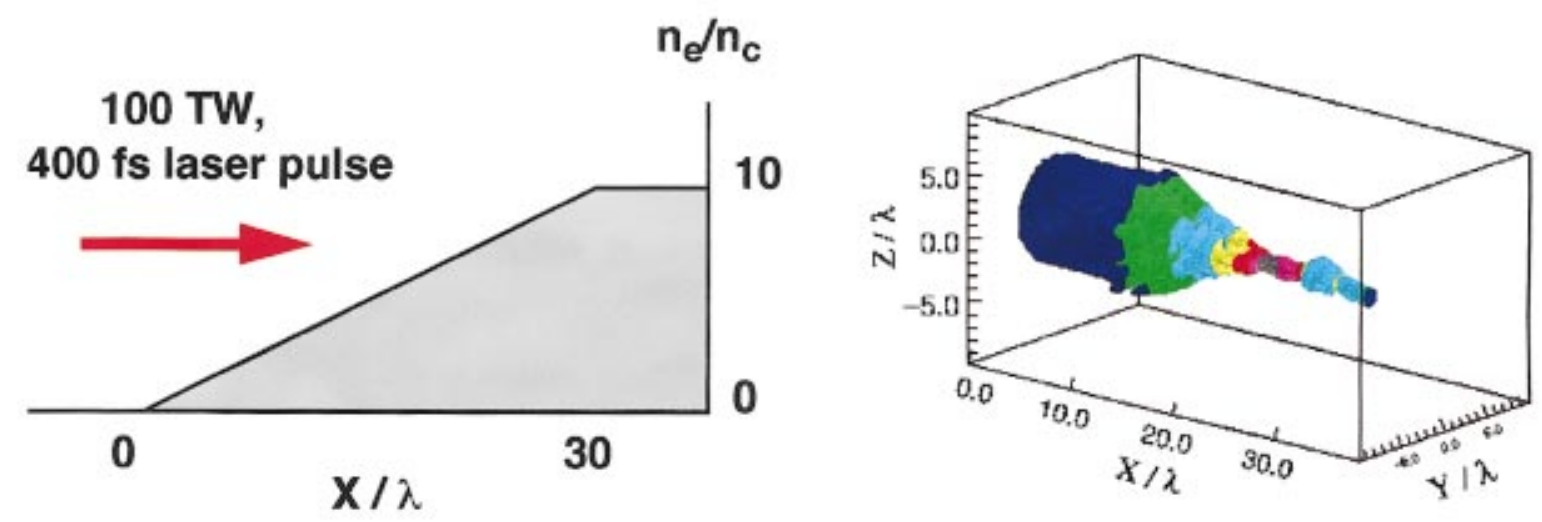

(b)

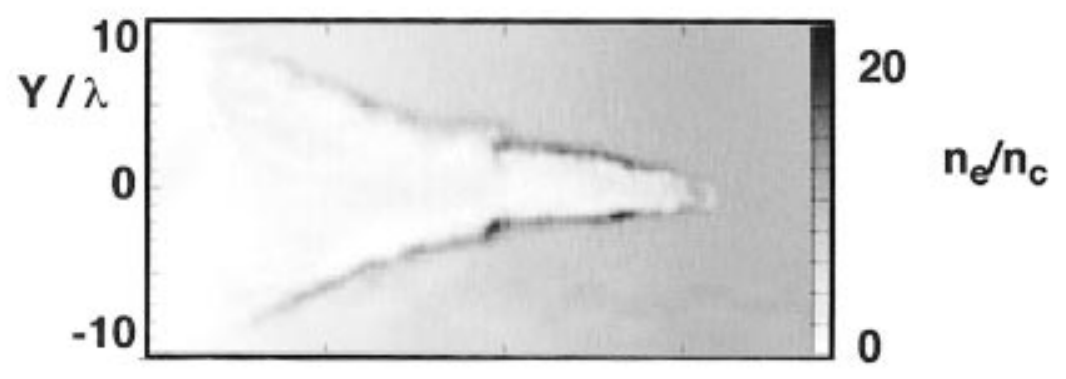

FIG. 2. (Color) 3D particle-in-call (PIC) simulation result of a super penetration shot. (a) Condition for the simulation, (b) electron density distribution.

carbon) targets. The focal positions vary from 100 to $600 \mu \mathrm{m}$ from the original target surface. The x-ray side-on pictures of those shots are shown in Fig. 1. Figures 1(a), 1(b), and 1(c) correspond to the focal positions of 100, 170, and $500 \mu \mathrm{m}$. In Figs. 1(a) and 1(c) there are large diameter emissions from the target surface, which come from the preproduced plasma created at a laser intensity $\left(I_{L}\right)$ of $10^{15} \mathrm{~W} / \mathrm{cm}^{2}$ within a 500 $\mu \mathrm{m}$ diam spot. There is a distinctive difference in Fig. 1(b) compared to the other two pictures. In Fig. 1(b) one can see a local emission of less than $30 \mu \mathrm{m}$ overlapped on the preproduced plasma almost on the target surface, as well as on the laser axis. In Fig. 1(c) a large x-ray emission is observed in the underdense plasma region. In our previous studies of ponderomotive type laser self-focusing, we have measured a whole beam self-focusing with various diagnostics. Those are $\mathrm{x}$-ray pinhole cameras, Doppler spectrum measurement in the backscattered laser light from the penetrating selffocusing head in the plasma, ${ }^{7}$ and ultraviolet (UV) (Ref. 8) and $\mathrm{x}$-ray laser ${ }^{9}$ probe beams. In these experiments, a $100 \mathrm{ps}$ pulse at $10^{17} \mathrm{~W} / \mathrm{cm}^{2}$ at $1 \mu \mathrm{m}$ was focused in a preproduced plasma at $250 \mu \mathrm{m}$ from the original target surface. We could show a very good correlation between the side-on x-ray pinhole pictures and whole beam self-focusing, which was monitored directly by the UV and $\mathrm{x}$-ray laser probes. Namely, when the laser light penetrate in a preproduced plasma as a whole beam self-focusing, and reached the sur- face of a planar target, a strong local x-ray emission of a 30 $\mu \mathrm{m}$ diameter was observed from both side and front directions equivalent to the focal spot in vacuum. In our $100 \mathrm{TW}$ laser experiment, the intense, locally emitted x-ray spot in Fig. 1(b) indicates that the ultra-intense laser light has penetrated in this thick preproduced plasma all the way, close to the target surface. There is another possibility to explain the local emission observed in Fig. 1(b), namely, by the hot electron themselves. However, the hot electron beam has a finite beam divergence angle, which is of the order of $30^{\circ} .{ }^{12}$ Considering the finite divergence angle of the hot electron beam, the observed local spot of about $30 \mu \mathrm{m}$ implies that the laser penetrated into the preproduced plasma and reached somewhere close to a point at $50 \mu \mathrm{m}$ from the target surface. A three-dimensional (3D) PIC simulation was run to study the self-focusing in a plasma with an exponential density profile. ${ }^{13}$ In the simulation a $100 \mathrm{TW}, 400$ fs laser pulse was focused down to $5 \times 10^{19} \mathrm{~W} / \mathrm{cm}^{2}$ incident on a plasma with a density rising from 0 to $10 n_{c}$ over $30 \mu \mathrm{m}$ and then remaining constant as shown in Fig. 2(a). The results are shown in Fig. 2(b) and 2(c). The laser self-focuses to an intensity of $10^{21} \mathrm{~W} / \mathrm{cm}^{2}$ in the channel and continues the propagation. In Fig. 2(b) the electron plasma density profile shows a clear channel formation, while the laser intensity distribution in Fig. 2(c) indicates a relativistic self-focusing. Such ultraintense laser propagates into the overdense plasma even if 


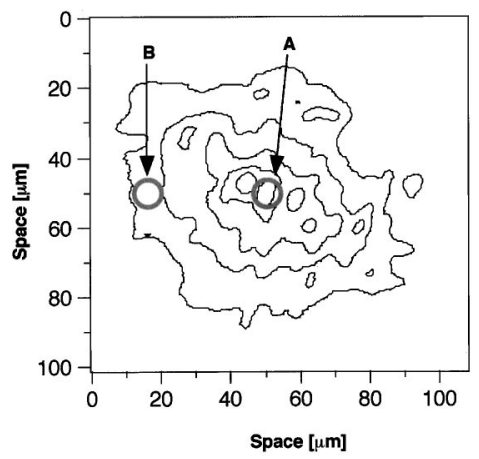

(a)

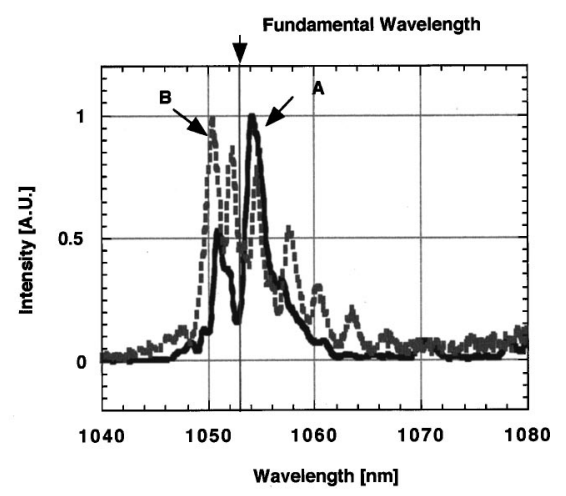

(b) the density is as high as 10 times $n_{c}$. A similar result has been obtained in our 2D PIC simulation.

Backscattered laser light from the preproduced plasma was measured with both spatial and spectral resolutions. ${ }^{14} \mathrm{~A}$ parabola focus mirror of F/3.5, which was used to irradiate the preproduced plasma, collected the backscattered light. The backscattered light was then image-relayed onto a bundle of 100 fibers (input) with a magnification of 15.4. The other side of the fiber bundle (output) was rearranged as a one-dimensional array of fibers. This array was attached on the entrance slit of a 1/4 m spectrometer. The exit slit image of the spectrometer shows the spectral dispersion in the horizontal direction and corresponds to each fiber in the vertical direction. Each vertical position corresponds to a part of focal area of $8 \mu \mathrm{m}$ diam. Figure 3(a) shows the image of the focal plane area in the plasma. The image was taken on the super penetrating shot of Fig. 1. A few intense hot spots are observed within the focal spot area. The spectra of one hot spot [A in Fig. 3(a)] and another part [B in Fig. 3(a)] in the weaker intensity region are shown in Fig. 3(b). The observed spectra are totally different between the hot spot and the other part within the focal area. This data indicates that the both spatial and spectral resolutions are necessary for spectral measurement of scattered light. The regularly spaced spectral peaks in Fig. 3(b) are unique and interesting. If we assume that the peaks are due to stimulated Brillouin scattering and its subsequent decay into several daughter waves, the spectral spacing between the first and second corresponds to $2 k_{L} C_{s}$, where $k_{L}$ is the pump laser wave number and $C_{s}$ $\left(=\left(T_{e} / M\right)^{1 / 2}\right)$ is the ion acoustic speed. Here $T_{e}$ is the plasma electron temperature and $M$ is the ion mass. Since $C_{s}$ is proportional to $\left(T_{e}\right)^{1 / 2}$, the background coronal temperature may be estimated to be about $6 \mathrm{keV}$.

\section{B. Hot electron generation}

On these super penetration shots, energetic electrons are measured both inside and outside the target. Electrons can be accelerated via $J \times B$ force ( $J$, electron current in the laser electric field and $B$, laser magnetic field), vacuum heating, and stimulated Raman scattering, etc. ${ }^{15}$ Electron spectrometers use a pair of magnet plates with a $1.2 \mathrm{kGauss}$ magnet strength. Since strong electromagnetic noise screens the most of the electrical diagnostics from 100 TW shots, an imaging plate $^{16}$ has been used as a detector. Figure 4 shows a typical hot electron spectrum measured at a $10^{19} \mathrm{~W} / \mathrm{cm}^{2}$ irradiation intensity for a case with the preproduced plasma. The horizontal axis shows the electron energy and the vertical shows the spectral intensity. The spectrum was measured at $19^{\circ}$ from the laser forward axis in the horizontal plane and $-45^{\circ}$ in the longitude. The peak of the spectrum is at around 1 $\mathrm{MeV}$. The spectrum shows hot electrons energetic enough for fast ignitor heating. ${ }^{1}$ The suggested temperature is about $2 \mathrm{MeV} .{ }^{12} K \alpha$ emission from a Ag signal layer was measured to estimate the effect of heating in a solid target via hot electrons. The target consists of $\mathrm{CD}$, Mo, and Ag layers, whose thickness are 30, 50-300, and $50 \mu \mathrm{m}$, respectively. The $100 \mathrm{TW}$ laser is irradiated from the CD side into the preproduced plasma at a laser intensity of $10^{19} \mathrm{~W} / \mathrm{cm}^{2}$. An electron beam with a spectrum shown in Fig. 4 goes into the target and cause $K \alpha$ emission in the Ag layer. The Mo layer was used as the absorbing layer of the electron beam. The $K \alpha$ emission in Ag becomes possible with energy larger than $25.5 \mathrm{keV}$. Figures 5(a) and 5(b) show the results of the $K \alpha$ emission measurement, where the horizontal axis is the photon energy and the vertical the photon intensity (arbitrary unit) with and without the preproduced plasma. The data were recorded with an x-ray charge coupled device (CCD) with a photon counting mode. The absolute sensitivity of the $\mathrm{CCD}$ was calibrated with ${ }^{55} \mathrm{Fe}\left(E_{K \alpha}=5.9 \mathrm{keV}\right)$ and

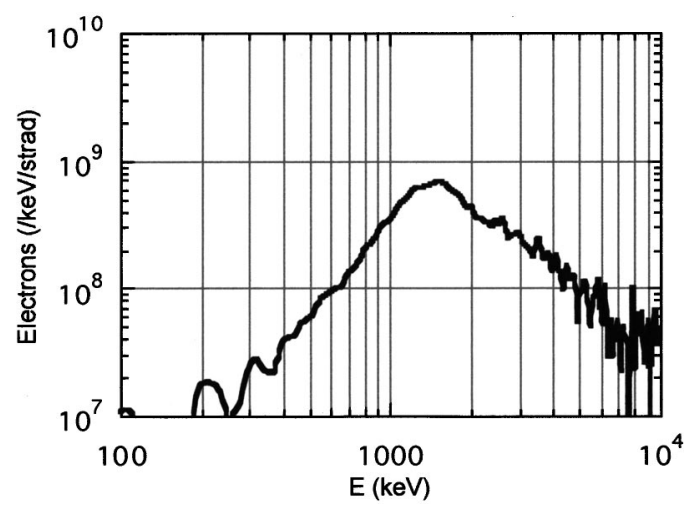

FIG. 4. Typical electron spectrum of ultra-intense laser shot into a preproduced plasma. 
(a) Without a preformed plasma

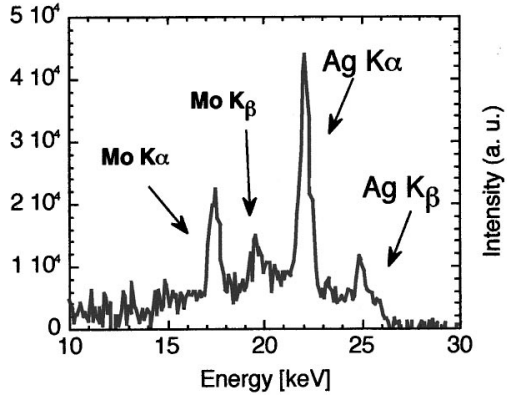

(b) With a preformed plasma

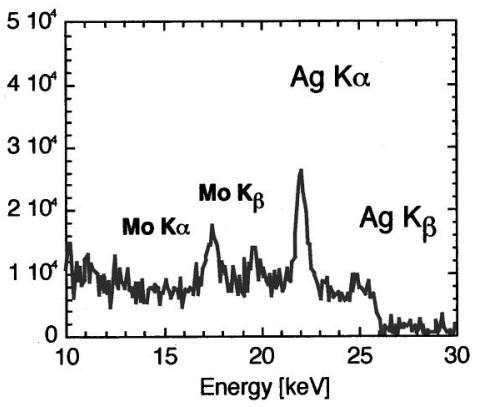

FIG. 5. $K \alpha$ emission spectra from (a) without plasma and (b) with a preproduced plasma. $K \alpha$ emissions from Ag signal layer were measured with an x-ray CCD.
${ }^{109} \mathrm{Cd}\left(E_{K \alpha}=22.2 \mathrm{keV}\right)$ and radiation sources. As shown in the figure, the level of $\mathrm{Ag} K \alpha$ emission is about half for a case with a preproduced plasma compared to a case without plasmas. This tendency is reflected to the conversion efficiency. The thickness of Mo absorption layer was varied to estimate the electron temperature from the decay slope of the emission with increasing thickness. The experimental data have been compared to the simulation by our 2D PIC code. The suggested temperature is about $2 \mathrm{MeV}$ and the conversion efficiency is $15 \%-20 \%$ for the case with the preproduced plasma.

\section{Energetic ions}

Ion acceleration may be possible subsequent to the hot electron generation. Once generated via ultra-intense laser, the ponderomotive force or quiver motion in the $e$-field hot electrons causes ion acceleration by the anomalous electrostatic field resulting from the charge separation. The mechanisms of the acceleration may be due to the electrostatic field created between the hot electrons and left over ions, ion reflection from the ion potential (Zagdeev potential) built up by the directly accelerated ions via the electrons, or Coulomb explosion. ${ }^{17-19}$ The acceleration direction of ions from the Coulomb explosion may be into $4 \pi$ steradian or normal to the surface of the explosion, while the first and second mechanisms may cause the forward-directed ions.

The ion acceleration has been studied via the fusion process, which is caused by fast deuterium ions from ultraintense laser plasma interactions colliding with deuterium within a solid target. Two time of flight detectors called "Mandala" were used as a single hit neutron detector with 421-elements each. ${ }^{20}$ The detector element was calibrated using accelerator facility. The energy resolution is $30 \mathrm{keV}$ for $2.5 \mathrm{MeV}$ neutrons. Each Mandala was placed at $54.7^{\circ}$ and $90^{\circ}$ from the $100 \mathrm{TW}$ laser axis, covering a solid angle of $20 \mathrm{msterad}$ with a $3 \mathrm{~m}$ diam. When a $\mathrm{CD}_{2}$ target of $60 \mu \mathrm{m}$ thickness was irradiated at a condition same as the super penetration shot described in Sec. II A, $2 \times 10^{5}$ DD neutrons are observed. More detailed studies of the neutron spectra have been conducted without preproduced plasmas, since less gamma ray noise is on the detectors without preproduced plasmas, resulting in much better $\mathrm{S} / \mathrm{N}$ of the signals. Figure 6 shows one of those neutron spectra (a) at $54.7^{\circ}$ and (b) $90^{\circ}$ from a CD planar target of $5 \mu \mathrm{m}$ irradiated at $10^{19} \mathrm{~W} / \mathrm{cm}^{2}$ with a possibly $10^{-4}$ prepulse level compared to the main laser intensity. When the accelerated ions move into a CD solid target, the fusion spectrum is affected according to the ion kinetic energy. The dominant process in our case is concluded to be due to $d^{*}(d, n)^{3} \mathrm{He}$, after several calibration shots carefully conducted. The neutron energy spectrum can be shifted from $1.63 \mathrm{MeV}$ to an upper limit determined by max ion energy. If the fusion process is exothermic and the spectrum is caused by a Doppler shift the neutron spectrum should be observed only above $1.63 \mathrm{MeV}$. The spectrum also depends sensitively on the observation angle (roughly with $\cos ^{2} \theta$ ). The neutron spectrum at $54.7^{\circ}$ in Fig. 6(a) extends from $2 \mathrm{MeV}$ to $4 \mathrm{MeV}$ peaked at just above $2.5 \mathrm{MeV}$. The one at $90^{\circ}$ in Fig. 6(b) extends similarly from $2 \mathrm{MeV}$ to $3.5 \mathrm{MeV}$. The peak is at just below 2.5 MeV. The number of neutrons is $1.0 \times 10^{6}$. The average energy of accelerated deuterons is estimated to be from $80 \mathrm{keV}$ to 100 $\mathrm{keV}$ when a Monte Carlo simulation is used to fit the observed data. The distribution of the deuteron acceleration into the target is within the forward $2 \pi$ hemisphere, slightly weighted toward the laser direction. Ion acceleration at a target backside has been studied using 2D (two-dimensional) PIC code simulation, as shown in Fig. 7. Here we note that the direction of the accelerated ions is normal to the target. In the simulation, a wedge shape target is used, whose sizes are $23 \mu \mathrm{m}$ the vertical direction and $13.5 \mu \mathrm{m}$ in the horizontal. The $100 \mathrm{TW}, 0.2$ ps laser pulse comes in from the left onto the target obliquely at $30^{\circ}$ with the $P$ polarization as shown in Fig. 7(a). The Roman number in Fig. 7(a) corresponds to the directions normal to each face of the wedge target. The target consists of electron plasma density of 4 times $n_{c}$. In Figs. 7(b) and 7(c) the accelerated ion flows are shown with a normalized momentum for both horizontal and vertical axes. Figures 7(b) and 7(c) correspond to time at 80 fs and 200 fs. Initially weak ion flows are observed normal to the target face normal directions. As time elapsed, much more energetic ion flows are observed normal to the target face as high as $4 \mathrm{MeV}$. Similar experimental results will be reported in Ref. 21, In the simulation hot electrons escaped from the target formed a electrostatic sheath, resulting in the ion acceleration normal to the target backside. Since the details of ion acceleration mechanisms will depend on the target thickness, target material, target shape, and laser polarization, further experimental studies will be called for. 

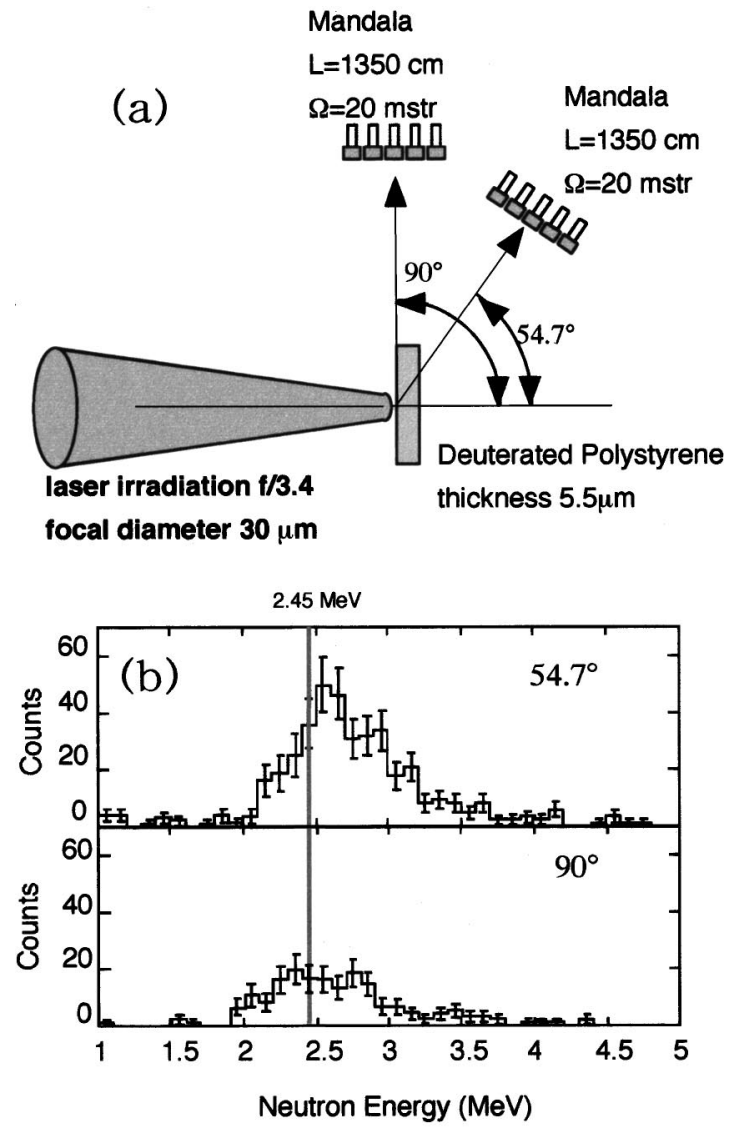

FIG. 6. Fusion neutron measurement with 842 channel single hit detectors "Mandala." (a) Experimental setup; (b) neutron signals at $54.7^{\circ}$ and $90^{\circ}$.

\section{First demonstration of a compressed core heated by short pulse self-focusing and hot electrons}

We have reported ponderomotive-force self-focusing at $1 \mu \mathrm{m}$ laser wavelength in plasmas with an overdense region with a $100 \mathrm{ps}$ pulse at a vacuum focused laser intensity of $10^{17} \mathrm{~W} / \mathrm{cm}^{2} .{ }^{7-9}$ We have learned that a whole beam selffocusing is formed in a plasma with a density scale length of $100 \mu \mathrm{m}$, producing hot electrons with a energy spectrum ranging from $100 \mathrm{keV}$ to $1 \mathrm{MeV}$. As a summary, the selffocusing occurs when $1 \mu \mathrm{m}$ laser is focused at close to the critical density in the preformed plasma at $10{ }^{17} \mathrm{~W} / \mathrm{cm}^{2}$ laser intensity. The penetration depth of the whole beam selffocusing can be more than $200 \mu \mathrm{m}$ in the plasma with an overdense region. Figure 8 summarizes our previous results. Figure 8(a) shows an interferogram picture of self-focused channel formation and cross sections in an underdense plasma. The interferogram was taken with an ultraviolet (UV) probe beam (wavelength, $\lambda=263 \mathrm{~nm}$; pulse width, $\tau=10 \mathrm{ps}$ ) to show a whole beam self-focusing. The selffocused channel in an overdense region was observed with an $\mathrm{x}$-ray laser probe $(\lambda=19.6 \mathrm{~nm}, \tau=80 \mathrm{ps})$ to show the whole beam self-focusing channel in Fig. 8(b), where the density profile and the cross section of the density profile are presented. In the density profile, a straight channel formation is clearly shown. In the cross section, two small arrows indicate the width of the channel formation of about $30 \mu \mathrm{m}$. Figure 8(c) shows a time-integrated $\mathrm{x}$-ray pinhole picture of (a)
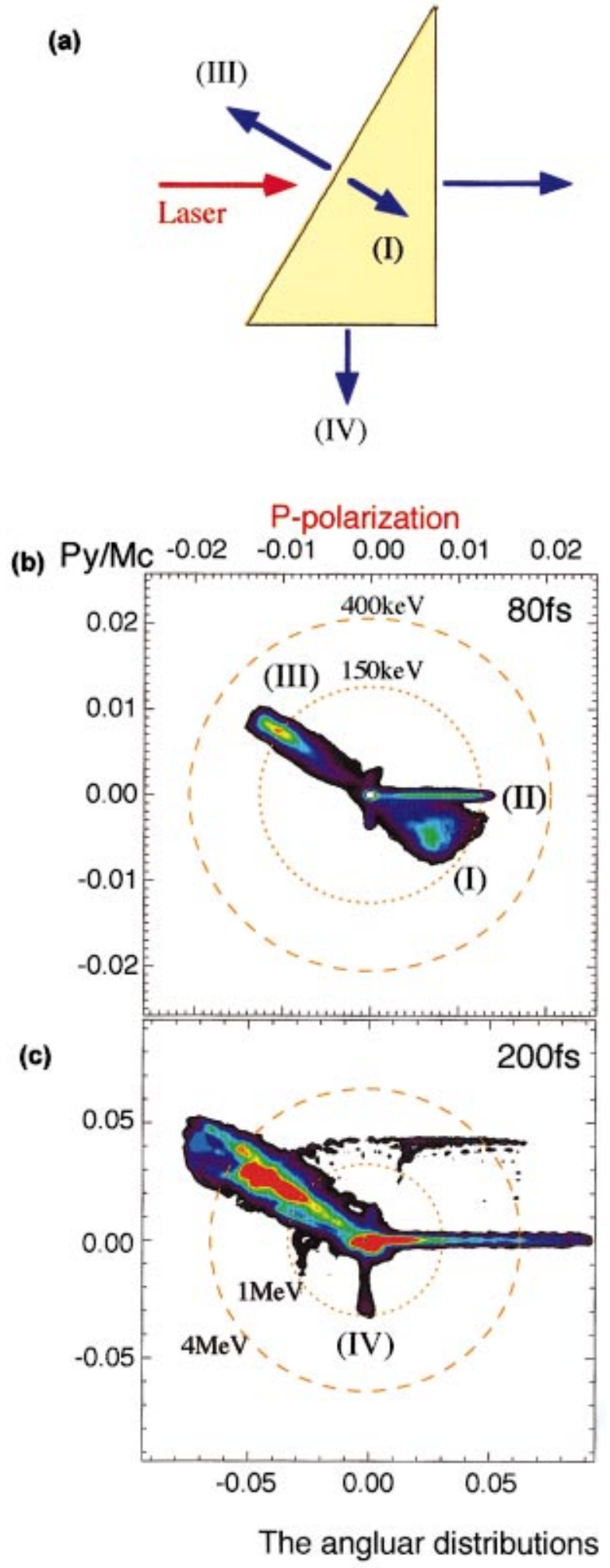

FIG. 7. (Color) Ions acceleration simulation for a wedge target. (a) Simulation set up, (b) ion acceleration at $80 \mathrm{fs}$, and (c) at $200 \mathrm{fs}$.

side and front views for the channel formation shot. The side-on x-ray picture shows a less than $30 \mu \mathrm{m}$ emission spot at the surface of a planar target, indicating that the selffocused laser beam penetrated through the preformed plasma and reached the surface region. The front view shows the same local emission due to the self-focused pulse. 


\section{Large scale plasma}

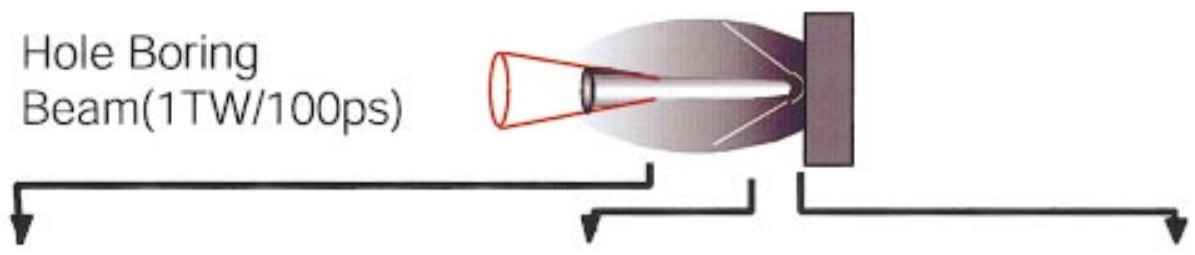

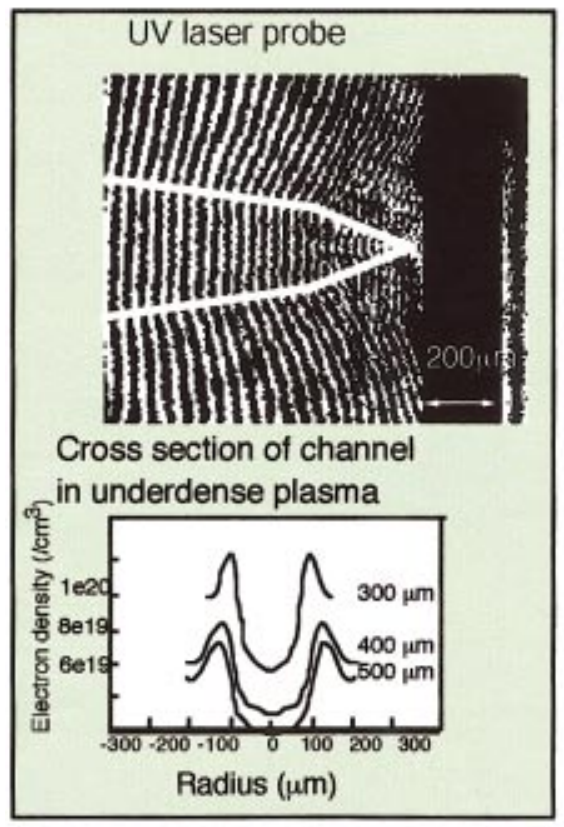

(a)

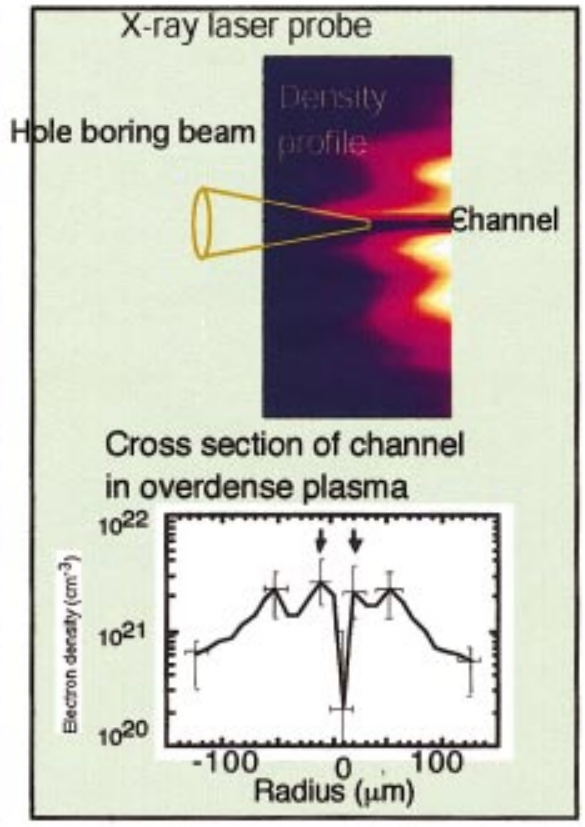

(b)

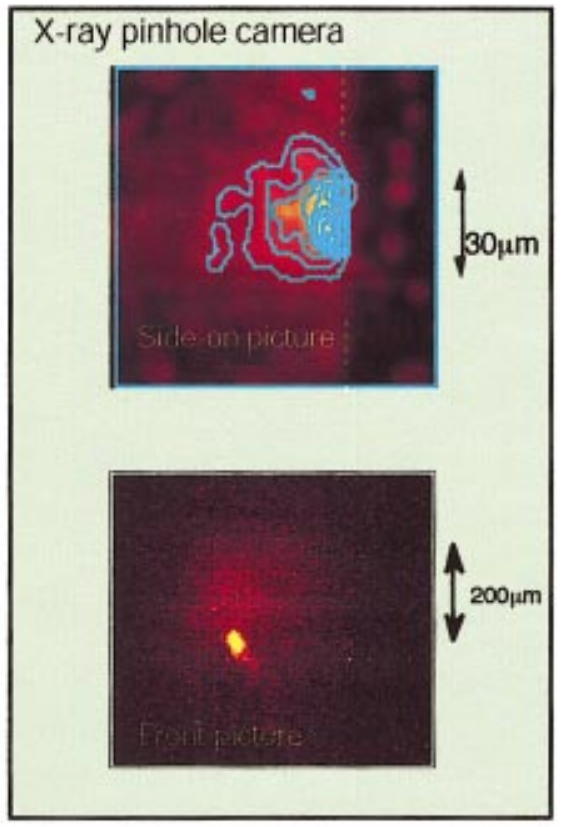

(c)

FIG. 8. (Color) Summary of whole beam channel in a plasma. (a) UV laser probe image and cross sections of self-focused channel in an underdense plasma; (b) x-ray laser probe image and cross sections of the channel in an overdense plasma, and (c) x-ray pinhole pictures of the channel.

Then we applied this type of self-focusing to an imploded core and tried to heat the core. It is very interesting to study how the whole beam self-focusing affects implosion cores or plasmas from the viewpoint of FI. In order to check this we have injected the 100 ps double pulses into implosion plasma. In order to satisfy the condition for a uniform implosion, we have to keep all twelve-laser beams of the GEKKO XII with a long pulse. For self-focusing and heating, we need laser beams with 100 ps pulses. The beam configuration applied in these shots is shown in Fig. 9. Each beam line of the GEKKO XII sends a 1 ns partially coherent (PCL) laser pulse followed by 100 ps double pulses with 150 ps time delay from the end of the PCL laser pulse. The 100 ps double pulses are separated by $300 \mathrm{ps}$. All the laser pulses are at $1 \mu \mathrm{m}$ wavelength. The PCL pulse is known to have a very uniform focused pattern ${ }^{22}$ and to have a large beam divergence angle $(120 \mu \mathrm{rad})$. Due to this beam divergence angle, the diameter of the far field pattern becomes $150 \mu \mathrm{m}$ for PCL, while the normal laser (100 ps pulse) focuses down to $30 \mu \mathrm{m}$. Making use of this focusing difference, the PCL pulse could be used for imploding a shell first and the laser pulses are used to self-focus and to heat the plasma. The CD shell of $193 \mu \mathrm{m}$ diam with a $21 \mu \mathrm{m}$ shell thickness was irradiated with the 12 beams of GEKKO XII. The total of $840 \mathrm{~J}$ PCL laser energy and $977 \mathrm{~J}$ first $100 \mathrm{ps}$ pulse and 742 $\mathrm{J}$ second 100 ps pulse are delivered onto the target. The laser beam focus was set at $170 \mu \mathrm{m}$ from the center of the target. ILESTA $^{23} 1 \mathrm{D}$ simulation result indicates that the density and $\rho R$ are $6 \mathrm{~g} / \mathrm{cm}^{3}$ and $0.018 \mathrm{~g} / \mathrm{cm}^{2}$ at the first $100 \mathrm{ps}$ injection and are $18 \mathrm{~g} / \mathrm{cm}^{3}$ and $0.041 \mathrm{~g} / \mathrm{cm}^{2}$, respectively, at the second. The core radius at the max compression (at the second $100 \mathrm{ps}$ timing) is $30 \mu \mathrm{m}$.

Figure 10 shows the $\mathrm{x}$-ray pinhole pictures of such shots. Figure 9(a) indicates a CD implosion result without 100 ps double pulses. The image shows weak x-ray emissions only from the initial shell diameter. The implosion is so weak that almost no x-ray emission comes from the core of $60 \mu \mathrm{m}$ diam. Figure 9(b) indicates a strong ringlike emission coming from roughly a $100 \mu \mathrm{m}$ diam. The additional $100 \mathrm{ps}$ pulses caused this strong emission. The double pulse emissions were confirmed clearly also with x-ray streak and x-ray framing cameras. On this shot hot electrons with an energy 


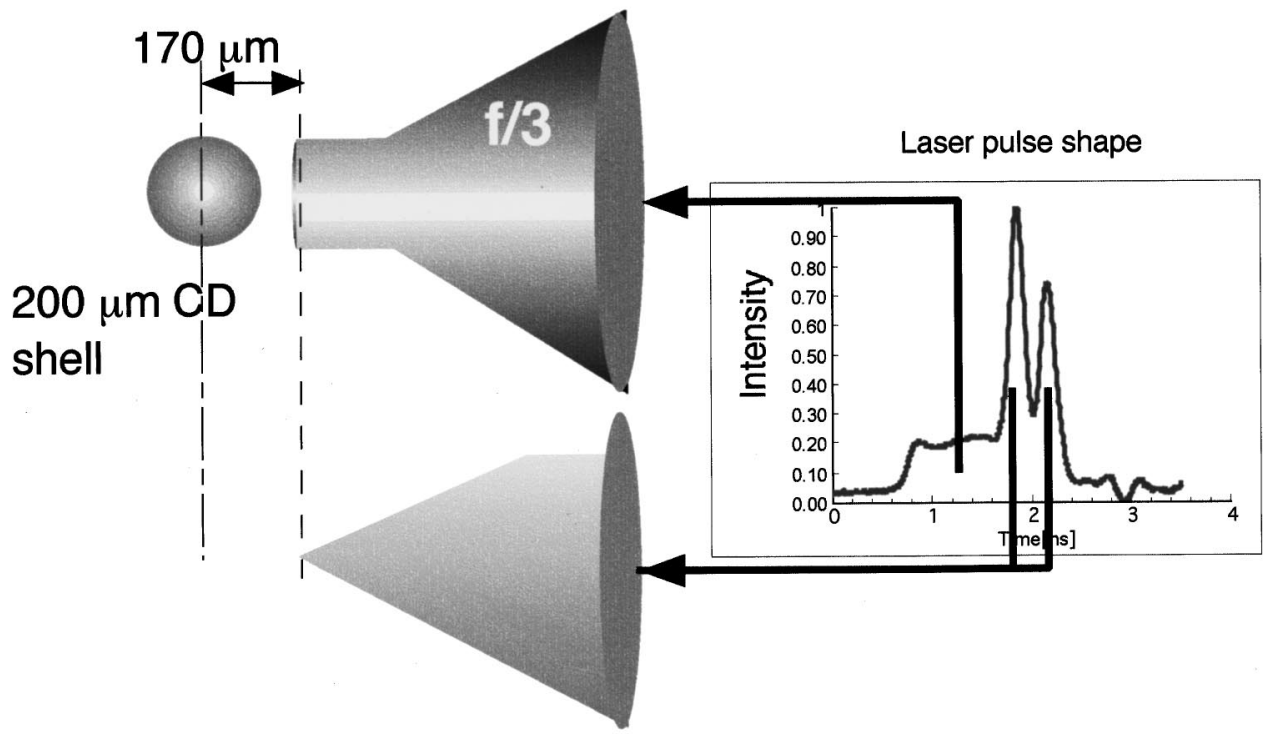

FIG. 9. Laser configuration used for implosion of a $\mathrm{CD}$ shell and subsequent core heating by $100 \mathrm{ps}$ double pulses. spectrum ranging from $100 \mathrm{keV}$ to $1 \mathrm{MeV}$ were also observed. This type of core heating will be an important subject for the near future experiments for FI and should be studied in detail. It should not be limited for fast ignition, such that a laser system in a reactor has only one fast ignition laser line. Each laser beam line can hold a long tailored pulse for implosion followed by short pulse(s) FI. Another advantage for multiple beams lines for fast ignition pulses could be that sending multiple pulses could avoid the Alfvén current limit and could transport a large amount of ignition energy to a imploded core.

\section{CONCLUSION}

We have shown the laser interaction studies with a 1 ps at $10^{19} \mathrm{~W} / \mathrm{cm}^{2}$ and a $100 \mathrm{ps}$ at $10^{17} \mathrm{~W} / \mathrm{cm}^{2}$ laser pulses. In the $10^{19} \mathrm{~W} / \mathrm{cm}^{2}$ experiments, a super penetration of laser pulse has been observed to penetrate in a plasma with a 100 $\mu \mathrm{m}$ density scale length and to reach the target surface. Over $1 \mathrm{MeV}$ energy electrons are observed and are converted to heat a solid target with a $20 \%$ efficiency with the presence of the plasma. Energetic deuterons are also produced to cause a $10^{6} \mathrm{D}-\mathrm{D}$ fusion product. The average accelerated ion energy is about $100 \mathrm{keV}$.

In the $10^{17} \mathrm{~W} / \mathrm{cm}^{2}$ experiment, a mode of whole beam self-focusing has been established in a preproduced plasma

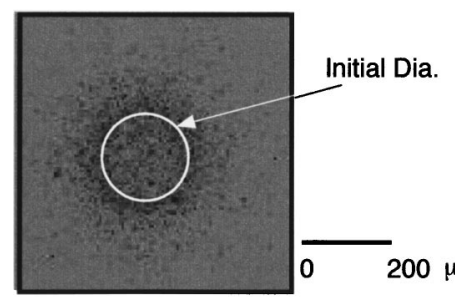

(a)

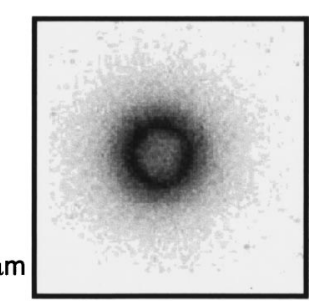

(b)
FIG. 10. X-ray pinhole pictures of CD shell implosion (a) without and (b) with self-focusing and heating pulses. The target diameter was $193 \mu \mathrm{m}$ with a $21 \mu \mathrm{m}$ shell thickness. The target was imploded with 12 infrared long pulses followed by two 100 ps pulses separated by 300 ps. and has been applied to an implosion plasma. The selffocused pulse could create $0.1-1 \mathrm{MeV}$ hot electrons. A weak and relatively large implosion core was created and was heated by the self-focusing and heating pulses for the first time.

\section{ACKNOWLEDGMENTS}

H. Asahara, H. Asamori, J. Hatano, C. Kamino, H. Kitamura, Y. Kimura, T. Kurita, T. Maeda, H. Matsuo, N. Morio, Dr. M. Nakai, Professor M. Nakatsuka, Dr. K. Nagai, M. Ohi, S. Okada, K. Sawai, K. Shimada, Ke. Suzuki, T. Sakamoto, T. Shimomura, R. Takahashi, S. Urushihara, N. Yamamoto, T. Yanagida, and K. Yosii are greatly acknowledged for their invaluable support in laser operation and target fabrication.

${ }^{1}$ M. Tabak, J. Hammel, M. Glinsky, W. L. Kruer, S. C. Wilks, J. Woodworth, E. M. Campbell, and M. Perry, Phys. Plasmas 1, 1626 (1996).

${ }^{2}$ H. Takabe, J. Plasma Fusion Res. 74, 316 (1998).

${ }^{3}$ D. Strickland and G. Mourou, Opt. Commun. 56, 219 (1985).

${ }^{4}$ M. Key, M. Cable, T. Cowan et al., Phys. Plasmas 5, 1996 (1998).

${ }^{5}$ A. J. Mackinnon, M. Borghesi, R. Gaillard, G. Malka, O. Willi, A. Offenberger, Poukov, J. Meyer-ter-Vehn, B. Canaud, J. L. Miquel, and N. B1anchot, Phys. Plasmas 6, 2185 (1999).

${ }^{6}$ J. Fuchs, P. C. Adam, F. Amiranoff, S. D. Baton, N. Banchot, P. Gallant, L. Gremillet, A. Heron, J. C. Kieffer, G. Laval, G. Malka, J. L. Miquel, P. Mora, H. Pepin, and C. Rousseaux, Phys. Plasmas 6, 2563 (1999).

${ }^{7}$ R. Kodama, K. Takahashi, K. A. Tanaka, M. Tsukamoto, H. Hashimoto, Y. Kato, and K. Mima, Phys. Rev. Lett. 77, 4906 (1996).

${ }^{8}$ K. A. Tanaka, H. Hashimoto, R. Kodama, K. Mima, Y. Sentoku, and K. Takahashi, Phys. Plasmas 60, 3283 (1999).

${ }^{9}$ K. Takahashi, R. Kodama, K. A. Tanaka, H. Hashimoto, Y. Kato, K. Mima, F. A. Weber, T. W. Barbee, Jr., and L. B. DaSilva, Phys. Rev. Lett. 84, 2405 (2000).

${ }^{10}$ A. Pukhov and J. Meyer-ter-Vehn, Phys. Plasmas 5, 1880 (1998); B. Lasinski, A. B. Langdon, S. P. Hatchett, M. H. Key, and M. Tabak, ibid. 6, 2041 (1999).

${ }^{11}$ J. Fuchs, G. Malka, J. C. Adams, F. Amiranoff, S. D. Baton, N. Blanchot, A. Heron, G. Laval, J. L. Miquel, P. Mora, H. Pepin, and C. Rosseaux, Phys. Rev. Lett. 80, 1658 (1998); J. Fuchs, J. C. Adamas, F. Amiranoff, S. D. Baton, P. Gallant, L. Gremillet, A. Heron, K. C. Kieffer, G. Laval, G. Malka, J. L. Miquel, P. Mora, H. Pepin, and C. Rosseuax, ibid. 80, 2326 (1998); V. Malka, E. D. Wispelace, F. Amiranoff, S. Baton, R. Bonadio, C. Coulaud, R. Haroutunian, A. Modena, D. Puissant, C. Stenz, S. Huller, and M. Casanova, ibid. 79, 2979 (1999). 
${ }^{12}$ R. Kodama, T. Matsusita, K. A. Tanaka et al., ILE Internal Report No. 9908, Institute of Laser Engineering, Osaka University (submitted to Phys. Rev. Lett.).

${ }^{13}$ A. Pukhov and J. Meyer-ter-Vehn, Phys. Plasmas 5, 1880 (1998).

${ }^{14}$ T. Miyakoshi, K. A. Tanaka, R. Kodama et al., Paper No. 3886-68 in SPIE Proceedings of Advanced High Power Lasers and Applications AHPLA '99, Nov. 1-5 (1999), Osaka, Japan (SPIE, Bellingham, WA) (to be published).

${ }^{15}$ S. C. Wilks and W. L. Kruer, IEEE J. Quantum Electron. 33, 1954 (1997).

${ }^{16}$ J. Miyahara, Solid State Phys. 30, 7 (1995) (in Japanese).

${ }^{17}$ Denavit, Phys. Rev. Lett. 23, 3052 (1992); S. Miyamoto, S. Kato, K. Mima, H. Takamura, R. Horiuchi, and T. Sato, J. Plasma Fusion Res. 73, 343 (1997); W. Yu, M. Y. Yu, J. Zhang, and Z. Xu, Phys. Rev. E 58, 6553 (1998).
${ }^{18}$ T. Dittmire, J. Zweiback, V. P. Yanovsky, T. E. Cowan, G. Hays, and K. B. Wharton, Nature (London) 398, 489 (1999).

${ }^{19}$ A. P. Fews, P. A. Norreys, F. N. Beg, A. R. Bell, A. E. Dangor, C. N. Danson, P. Lee, and S. L. Rose, Phys. Rev. Lett. 73, 1801 (1994); L. Disdier, J.-P. Garconnet, G. Malka, and J-L. Miquel, ibid. 82, 454 (1999).

${ }^{20}$ N. Izumi, K. Yamaguchi, T. Yamagajo et al., Rev. Sci. Instrum. 70, 1221 (1999).

${ }^{21}$ M. Key, Paper No. Mola1-327, in Proceedings of the First Inertial Fusion Sciences and Applications (IFSA), Bordeaux, France, Sept. 13-18, (1999) (Elsevier, New York) (to be published); T. Cowan (private communications, 1999).

${ }^{22}$ H. Nakano, T. Tsubakimoto, N. Miyanaga, M. Nakatsuka, T. Kanabe, H. Azechi, T. Jitsuno, and S. Nakai, J. Appl. Phys. 73, 2122 (1993).

${ }^{23}$ H. Takabe, Phys. Fluids 31, 2284 (1998); H. Takabe and T. Ishil, Jpn. J. Appl. Phys. 32, 5675 (1993). 